\title{
Primary Care Equals Secondary Prevention in Ischemic Heart Disease
}

\author{
Gbenga Ogedegbe, MD and Stephen K. Williams, MD \\ Center for Healthful Behavior Change, Division of General Internal Medicine, Department of Medicine, New York University School of \\ Medicine, New York, NY, USA.
}

J Gen Intern Med 26(10):1086-7

DOI: $10.1007 / \mathrm{s} 11606-011-1823-5$

(C) Society of General Internal Medicine 2011

$\mathrm{T}$ he prevalence of ischemic heart disease (IHD) is high among the elderly. Current projections indicate an even higher IHD prevalence over the next decade in the United States with significantly higher costs to an already taxed healthcare system. ${ }^{1}$ Accompanying this increasing burden of IHD is an undersupply of cardiovascular disease (CVD) specialists, ${ }^{1}$ meaning that primary care providers (PCPs) will remain the first line of care for patients with IHD in the foreseeable future.

In this issue of the Journal, the study by Einarsdottir et al. demonstrates the benefits of regular PCP follow-up in elderly patients discharged with a diagnosis of IHD. ${ }^{2}$ In a retrospective cohort study among over 30,000 elderly (mean age 73 years) discharged with IHD in Western Australia (WA), the authors evaluated the effect of regular primary care follow-up [postdischarge] on all-cause mortality and repeat hospitalization rates. For this purpose, the authors linked the WA Department of Health database with hospital, mortality, health insurance, and residency databases. The period following the index hospitalization event was divided into a post-discharge 'exposure' period during which the primary care regularity score was assessed and a 'follow-up' period during which the censoring event was recorded. Cases of index IHD hospitalization were ascertained using hospital administrative discharge codes. Frequency of visits was recorded during the 'exposure' period using health insurance databases that record outpatient events. A patient was censored at the time of death or time of IHD re-hospitalization, whichever occurred first. In order to minimize reverse causation bias that can be observed with increased outpatient utilization accompanying clinical deterioration preceding repeat hospitalization and/or death, the authors instituted a six-month 'wash out' period between the exposure and follow-up period; and no exposures or outcomes were ascertained during this period. The authors found a doseresponse relationship between primary care regularity and risk of repeat hospitalization and mortality. Specifically, the quartile that represented the group with the most regular follow-up had improved hazard ratios (HR) for all-cause and IHD mortality (HR 0.71 [CI: 0.63-0.82] and HR 0.65 [CI: 0.51-0.83], respectively). This study has three important strengths. First,

Published online August 12, 2011 the authors used a novel measure of primary care regularity that was developed by the same group with proven validity in other patient populations. ${ }^{3,4}$ Second, they applied a washout period to reduce the impact of the often-common bias of confounding by indication that plagues this kind of study. Third, and most important, this is the first study to use population level data to assess the benefit of primary care regularity in patients with a history of hospitalization for IHD. The investigators were able to accomplish this feat because the Western Australian Department of Health collects administrative data on the entire population; thereby avoiding the inherent selection bias that can occur in cohort studies when there is a selection of a representative sample from a larger population of interest.

Findings from the Einarsdottir et al. study raise two interesting questions. First, what are the potential mechanisms through which primary care regularity affects hospitalization and mortality in IHD patients? In an attempt to address the question, the authors suggest that primary care regularity may be associated with increased adherence to prescribed medications and improved provider-patient relationships. We can derive some insight in this regard from the Irish Heartwatch experience in which a program was initiated to address the continuity of care for patients with IHD. ${ }^{5}$ This program, which provided patients up to four PCP visits per year, was associated with a significant increase in the prescription of statins, angiotensin converting enzyme inhibitors, and beta-blockers and a resultant improvement in blood pressure control, lipid profiles and smoking cessation rates. ${ }^{5}$ It should be noted that there is a striking difference between the four PCP visits per year described in the Heartwatch program and the mean of ten PCP visits per year noted in the Einarsdottir article.

Second, what are the policy implications of these findings for the current health care reform debate in the United States? We argue that this Australian study is a timely addition to the literature in this regard. The Patient Protection and Affordable Care Act (PPACA) was formulated upon an expanded role for PCPs in the care of patients with chronic diseases. ${ }^{6}$ An increased PCP role is desperately needed for widespread implementation of current guidelines for management of patients with CVD. The American Heart Association /American College of Cardiology (AHA/ACC) recommends that all medical care settings in which IHD patients are encountered should have a system in place to encourage guideline implementation, ${ }^{7}$ which advocate a comprehensive risk factor management including lifestyle modification, lipid, diabetes, and blood pressure control, and an aggressive use of anti-platelet therapy, beta-blockers, statins and Renin Angiotensin Aldosterone System blockers. Such comprehensive management of risk factors requires regular follow-up with PCPs. 
Unfortunately, the most recent update of the AHA/ACC guidelines lamented that patients were still not getting evidence-based guideline-concordant treatment. ${ }^{8}$ Thus a healthcare reform without an adequate primary care base certainly cannot deliver recommended guidelines for secondary prevention of CVD in a cost-effective manner. The findings from this study are indeed very encouraging, and indicate that we are on the right path in insisting that health reform provide guarantees of primary care for all patients with chronic diseases. The recent growth of the Patient Centered Medical Home model, which is aimed at facilitating patient access to primary care services, is a right step in this direction. Similarly, the establishment of the PatientCentered Outcomes Research Institute would facilitate the evaluation of evidence-based comparative effectiveness studies that would allow policy makers to determine exactly how often, and how much primary care is needed for patients afflicted with IHD - still the most common cause of death in the US.

Despite the encouraging findings from this study, future research should replicate this study in the US with more representative populations to address the following questions: what is the optimal frequency of PCP visits? At what costs to the health care system do these closer follow-up visits come? What are the specific mechanisms for the effect of continuity of care on important health outcomes and resource utilization? What role do health information technology systems play in facilitating coordination of care in post-discharge patients, and are these cost-effective? These issues should be at the forefront of leading health policy institutions in the US, as we strive to implement population-based interventions that can help to achieve improved outcomes in patients with IHD.

Acknowledgements: Dr. Williams was supported in part by grants from the Agency for Healthcare Research and Quality T32 HS 000066.
Dr. Ogedegbe was supported in part by grants: R01HL087301 and R01HSO18589

Corresponding Author: Gbenga Ogedegbe, MD; Center for Healthful Behavior Change, Division of General Internal Medicine, Department of Medicine, New York University School of Medicine, 227 East 30th Street (between 2nd and 3rd Avenues), 6th Floor, New York, NY 10016, USA (e-mail: olugbenga.ogedegbe@nyumc.org).

\section{REFERENCES}

1. Heidenreich PA, Trogdon JG, Khavjou OA, et al. Forecasting the future of cardiovascular disease in the United States: a policy statement from the American Heart Association. Circulation. 2011;123:933-944.

2. Einarsdottir K, Preen DB, Emery JD, Holman CD. Regular primary care plays a significant role in secondary prevention of ischemic heart disease in a Western Australian cohort. J Gen Intern Med. doi:10.1007/s11606011-1665-1.

3. Einarsdottir K, Preen DB, Emery JD, Kelman C, Holman CD. Regular primary care lowers hospitalisation risk and mortality in seniors with chronic respiratory diseases. J Gen Intern Med. 2010;25:766-773.

4. Einarsdottir K, Preen DB, Emery JD, Holman CD. Regular primary care decreases the likelihood of mortality in older people with epilepsy. Med Care. 2010;48:472-476.

5. Fitzpatrick P, Fitz-Simon N, Lonergan M, Collins C, Daly L. Heartwatch: the effect of a primary care-delivered secondary prevention programme for cardiovascular disease on medication use and risk factor profiles. Eur J Cardiovasc Prev Rehabil. 2011;18:129-135.

6. Goodson JD. Patient protection and affordable care act: promise and peril for primary care. Ann Intern Med. 2010;152:742-744.

7. Smith SC Jr, Blair SN, Bonow RO, et al. AHA/ACC scientific statement: AHA/ACC guidelines for preventing heart attack and death in patients with atherosclerotic cardiovascular disease: 2001 update: a statement for healthcare professionals from the American Heart Association and the American College of Cardiology. Circulation. 2001;104:1577-1579.

8. Smith SC Jr, Allen J, Blair SN, et al. AHA/ACC guidelines for secondary prevention for patients with coronary and other atherosclerotic vascular disease: 2006 update endorsed by the National Heart, Lung, and Blood Institute. J Am Coll Cardiol. 2006;47:2130-2139. 